\title{
PROPAGATION PROPERTIES IN SCATTERING THEORY
}

\author{
DEREK W. ROBINSON
}

(Received 14 June 1979)

(Revised 6 September 1979)

\begin{abstract}
Generalizations of the Green-Lanford-Dollard theorem on scattering into cones and the Ruelle-Amrein-Georgescu theorem characterizing bound states and scattering states are derived. The first is shown to be an easy consequence of the Kato-Trotter theorem on semi-group convergence whilst the latter is a corollary of Wiener's version of the mean ergodic theorem.
\end{abstract}

\section{Introduction}

Scattering theory consists of a quantitative comparison of the asymptotic motion of an interacting particle system with the corresponding motion of a non-interacting system. This comparison is facilitated by a priori estimates on both types of motion and such estimates are the subject of the present note.

In two-particle quantum mechanical scattering theory the Green-Lanford [4] and Dollard [3] theorem (the GLAD theorem) provides basic information on the free propagation necessary for the asymptotic particle description. This theorem states that the probability that a non-interacting particle is eventually in a cone $C$ with apex at the origin, that is,

$$
C=\{x ; x . n \geqslant \alpha|x|,|n|=1,0<\alpha \leqslant 1\},
$$

is equal to the probability that the particle velocity lies in $C$. We generalize this result by demonstrating, for a quasi-free evolution with energy momentum relation $p \rightarrow \omega(p)$, that the probability the particle is in the set $t S$ at time $t$ is asymptotically equal to the probability that the generalized velocity $\nabla \omega(p)$ lies in $S$. ( $S$ is a bounded open set in the configuration space $\mathbf{R}^{\nu}$.)

The Ruelle [9] and Amrein-Georgescu [1] theorem (the RAG theorem) concerns the relative motion of two interacting particles and characterizes states of the point 
spectrum and states of the continuous spectrum in terms of spatial probability distributions. To be more precise, let $\psi \rightarrow \psi_{t}=e^{i t H} \psi$ describe the evolution of $\psi \in L^{2}\left(\mathbf{R}^{v}\right)$ corresponding to the relative motion given by the self-adjoint Hamiltonian $H$ and define $M_{\psi}$ by

Clearly

$$
M_{\psi}=\lim _{R \rightarrow \infty} \sup \lim _{T \rightarrow \infty} \sup \frac{1}{T} \int_{0}^{T} d t \int_{|x| \leqslant R} d^{v} x\left|\psi_{t}(x)\right|^{2} /\|\psi\| \|^{2} .
$$

$$
0 \leqslant M_{\psi} \leqslant 1 \text {, }
$$

and $\mathrm{M}_{\psi}$ is a crude measure of the proportion of the time the particles are close together. Under weak assumptions on the particle interaction we demonstrate $\psi$ is in the subspace $\mathscr{H}_{p}$ corresponding to the point spectrum of $H$ if, and only if, $M_{\psi}=1$, whilst $\psi$ is in the subspace $\mathscr{H}_{c}$ of continuous spectrum if, and only if, $M_{\psi}=0$. (In fact we derive an exact expression for $M_{\psi}$ in terms of the eigenprojections of $H$.) This characterization of $\mathscr{H}_{c}$ is exactly that given by the RAG theorem but the condition $M_{\psi}=1$ is a stronger criterion for $\psi$ to be in $\mathscr{H}_{p}$ than that of the RAG result.

These generalizations of the GLAD-RAG theorems should also serve a pedagogic purpose. Whilst previous derivations of the GLAD theorem depended upon special choices of $\omega(p)$, for example, the non-relativistic form $\omega(p)=p^{2}$, see [3, 4], or special properties of $\omega(p)$, for example, monotonicity in $|p|$, see [5], the present proof relies solely on the Kato-Trotter theorem for the convergence of perturbed unitary groups. Our proof of the RAG theorem is also conceptually simpler than the original proofs insofar as it uses only the mean ergodic theorem.

\section{The GLAD theorem}

Elements $\phi, \psi, \ldots$ of the Hilbert space $L^{2}\left(\mathbf{R}^{v}\right)$ can be thought of as representing states of a quantum mechanical particle in the configuration space $\mathbf{R}^{v}$ or the states of relative motion of two such particles. These two points of view lead to identification of the dynamical problem of a particle moving in a potential field and the problem of the relative motion of two particles with a potential interaction. For simplicity we adopt the first viewpoint and subsequently talk about the motion of a single particle.

Define a one-parameter group of unitary maps on $L^{2}\left(\mathbf{R}^{\eta}\right)$ by

$$
\psi(x) \rightarrow \psi_{t}(x)=(2 \pi)^{-\mathrm{v} / 2} \int d^{\mathrm{v}} p \tilde{\psi}(p) e^{i p x-\omega(p) t},
$$

where $\tilde{\psi} \in L^{2}\left(\mathbf{R}^{\eta}\right)$ denotes the Fourier transform of $\psi \in L^{2}\left(\mathbf{R}^{\eta}\right)$ and $\omega \in C(\mathbf{R})$. This group describes the evolution of a particle whose energy $\omega$ is determined com- 
pletely by its momentum $p$. We refer to it as a quasi-free evolution. The choice $\omega(p)=p^{2} /(2 m)$ corresponds to a non-relativistic particle of mass $m$ and in this case the Green-Lanford-Dollard theorem [3, 4] states that

$$
\lim _{t \rightarrow \infty} \int_{x \in C} d^{v} x\left|\psi_{\imath}(x)\right|^{2}=\int_{p / m \in C} d^{v} p|\tilde{\psi}(p)|^{2},
$$

where $C$ is any cone with apex at the origin. This theorem has been extended to quasi-free evolutions for which $\omega$ is an increasing, differentiable, function of $|p|$ by Jauch et al. [5]. Further discussion of the physical interpretation of the theorem can be found in $[2,7]$.

Our first result gives a generalization to quasi-free evolutions for which $\omega$ is once-continuously differentiable.

THeOREM 1. Let $\psi \in L^{2}\left(\mathbf{R}^{v}\right) \rightarrow \psi_{t} \in L^{2}\left(\mathbf{R}^{v}\right)$ denote the quasi-free evolution corresponding to $\omega \in \mathbf{C}^{1}\left(\mathbf{R}^{\eta}\right)$. Then

$$
\lim _{|t| \rightarrow \infty} \int d^{v} x\left|\psi_{t}(x)\right|^{2} f(x / t)=\int d^{v} p|\tilde{\psi}(p)|^{2} f(\nabla \omega(p))
$$

for every bounded continuous $f$ on $\mathbf{R}^{v}$. Consequently

$$
\lim _{|t| \rightarrow \infty} \int_{x / t \in S} d^{v} x\left|\psi_{t}(x)\right|^{2}=\int_{\nabla \omega(p) \in S} d^{v} p|\psi(p)|^{2}
$$

for each bounded, open, set $S \subset \mathbf{R}^{v}$ such that $\{p ; \nabla \omega(p) \in \bar{S} \backslash S\}$ has Lebesgue measure zero.

Proof. First define $X=\left(X_{1}, \ldots, X_{\mathrm{v}}\right)$ as the operators of multiplication, $\left(X_{i} \psi\right)(x)=x_{i} \psi(x)$, and define $P=\left(P_{1}, \ldots, P_{v}\right)$ as the differential operators which correspond to multiplication of the Fourier transform, that is, $\left(P_{i} \tilde{\psi}\right)(p)=p_{i} \tilde{\psi}(p)$. The domains of these operators are specified by

$$
D\left(X_{i}\right)=\left\{\psi ; \int d^{v} x\left(1+\left|x_{i}\right|^{2}\right)|\psi(x)|^{2}<+\infty\right\}
$$

and

$$
D\left(P_{i}\right)=\left\{\psi ; \int d^{v} p\left(1+\left|p_{i}\right|^{2}\right)|\tilde{\psi}(p)|^{2}<+\infty\right\},
$$

and both operators have self-adjoint components. Note that the evolution $\psi \rightarrow \psi_{\text {t }}$ can be written

where

$$
\psi_{t}=U_{t} \psi
$$

$$
U_{t}=\exp \{-i t \omega(P)\}
$$


Next introduce the one-parameter family of "velocity" operators

$$
V_{t}=U_{-t} X U_{t} / t
$$

The components of $V_{t}$ are self-adjoint on $U_{-t} D\left(X_{i}\right)$. But the set $D$ formed by the $\psi$ whose Fourier transforms $\tilde{\psi}$ are once-differentiable with compact support is a core for each $X_{i}$ and $U_{t} D=D$. Hence $D$ is a core for the components of each $V_{i}$. Moreover, if $\psi \in D$, one easily computes that

$$
V_{t} \psi=X \psi / t+\nabla \omega(P) \psi
$$

Consequently

$$
\lim _{|t| \rightarrow \infty}\left\|\left(V_{t}-\nabla \omega(P)\right)_{i} \psi\right\|=0
$$

for all $\psi$ in the common core $D$. But this implies that $V_{t}$ converges to $\nabla \omega(P)$ componentwise in the strong generalized sense (see, for example, [6], Chapter VIII and in particular Section 1.1, Corollary 1.6). But the components of $V_{t}$ mutually commute as do the components of $\nabla \omega(P)$. Thus the unitary group $\exp \left\{i s . V_{t}\right\}$ converges strongly to the unitary group $\exp \{i s . \nabla \omega(P)\}$ as $|t| \rightarrow \infty$. Moreover $f\left(V_{t}\right) \rightarrow f(\nabla \omega(P))$ for each bounded continuous $f$ (see, for example, [8], Theorem VIII, 20(6)). Therefore

$$
\begin{aligned}
\lim _{|t| \rightarrow \infty} \int d^{v} x\left|\psi_{t}(x)\right|^{2} f(x / t) & =\lim _{|t| \rightarrow \infty}\left(\psi, U_{-t} f(X / t) U_{t} \psi\right) \\
& =\lim _{|t| \rightarrow \infty}\left(\psi, f\left(V_{t}\right) \psi\right) \\
& =(\psi, f(\nabla \omega(P)) \psi) \\
& =\int d^{v} p|\psi(p)|^{2} f(\nabla \omega(P)) .
\end{aligned}
$$

Now the final statement of the theorem follows formally from the first statement by choosing $f$ to be the characteristic function $\chi_{S}$ of the set $S$. But since this latter function is not continuous it is necessary to use an approximation procedure. One chooses two sequences of bounded continuous functions $f_{n}, g_{n}$, such that $f_{n} \geqslant \chi_{s}, g_{n} \leqslant \chi_{s},\left|f_{n}\right|,\left|g_{n}\right| \leqslant 1$, and $f_{n} \rightarrow \chi_{S}, g_{n} \rightarrow \chi_{s}$ pointwise. Consequently

$$
\begin{aligned}
\lim _{|t| \rightarrow \infty} \sup \int_{x / t \in S} d^{v} x\left|\psi_{t}(x)\right|^{2} & \leqslant \lim _{|t| \rightarrow \infty} \int d^{v} x\left|\psi_{t}(x)\right|^{2} f_{n}(x / t) \\
& =\int d^{v} p|\psi(p)|^{2} f_{n}(\nabla \omega(p)) \\
& \underset{n=\infty}{\longrightarrow} \int_{\nabla \omega(p) \in S} d^{v} p|\psi(p)|^{2},
\end{aligned}
$$


where the last step uses Lebesgue's dominated convergence theorem. Similarly,

$$
\begin{aligned}
\lim _{|t| \rightarrow \infty} \inf \int_{x / t \in S} d^{v} x\left|\psi_{t}(x)\right|^{2} & \geqslant \int d^{v} p|\tilde{\psi}(p)|^{2} g_{n}(\nabla \omega(p)) \\
& \underset{n=\infty}{\longrightarrow} \int_{\nabla \omega(p) \in S} d^{v} p|\psi(p)|^{2} .
\end{aligned}
$$

The desired result follows by combination of these statements.

The second statement of the theorem gives a probabilistic interpretation of $\nabla \omega(p)$ as the asymptotic value of $x / t$, that is, the particle velocity. It states that for large times $t$ the probability that the velocity $x / t$ is in $S$ is the same as the probability that $\nabla \omega(p) \in S$.

One interesting case for which the theorem applies is that of a relativistic particle of mass $m$. Thus $\omega(p)=\sqrt{ }\left(p^{2}+m^{2}\right)$ if the speed of light is set equal to one. Hence $\nabla \omega(p)=p / \sqrt{ }\left(p^{2}+m^{2}\right)$ and, in particular, $|\nabla \omega(p)| \leqslant 1$. Consequently the theorem implies that

$$
\lim _{|t| \rightarrow \infty} \int_{|x|>|t|} d^{v} x\left|\psi_{t}(x)\right|^{2}=0,
$$

that is, asymptotically the particle is localized within the light cone.

The result of the theorem is easily interpretable in classical mechanics. The classical orbit is given by $x=\nabla \omega(p) t+a$, where $a$ is the position at time zero, and hence

$$
f(x / t)=f\left(\nabla \omega(p)+\frac{a}{t}\right) \rightarrow f(\nabla \omega(p))
$$

for each continuous $f$. If, however, $f$ is twice differentiable one obtains the more detailed estimate

$$
f(x / t)=f(\nabla \omega(p))+\frac{a}{t} f^{\prime}(\nabla \omega(p))+O\left(t^{-2}\right) .
$$

Thus the $O\left(t^{-1}\right)$ term is proportional to the original displacement. Under more stringent regularity properties of $f, \psi$ and $\omega$, one may obtain similar estimates in the quantum case. The simplest method is to assume $f \in L^{1}\left(\mathbf{R}^{v}\right)$, to write

$$
\begin{aligned}
\int d^{v} x\left|\psi_{t}(x)\right|^{2} f(x / t) & =\left(\psi, f\left(V_{t}\right) \psi\right) \\
& =(2 \pi)^{-v / 2} \int d^{v} s f(s)\left(\psi, \exp \left(i s V_{t}\right) \psi\right),
\end{aligned}
$$

and then to use perturbation theory on the group $\exp \left(i s V_{t}\right)$. 
Alternatively, one may obtain generalizations $[3,4]$ to other evolution groups incorporating a potential interaction with the aid of the Møller matrices. Let $\hat{U}_{t}$ be a one-parameter group on $L^{2}\left(\mathbf{R}^{v}\right)$ such that the Møller matrices

$$
\Omega_{ \pm} \psi=s . \lim _{t \rightarrow \pm \infty} \hat{O}_{-t} U_{t} \psi
$$

with respect to the quasi-free group exist. These operators are isometric and invertible from the ranges $R\left(\Omega_{ \pm}\right)$to $L^{2}\left(\mathbf{R}^{v}\right)$. Moreover, if $\psi \in R\left(\Omega_{ \pm}\right)$

Thus, for $\psi \in R\left(\Omega_{ \pm}\right)$,

$$
\Omega_{ \pm}^{-1} \psi=s . \lim _{t \rightarrow \pm \infty} U_{-t} \hat{O}_{t} \psi
$$

$$
\begin{aligned}
\lim _{t \rightarrow \pm \infty} \int d^{v} x\left|\left(O_{t} \psi\right)(x)\right|^{2} f(x / t) & =\lim _{t \rightarrow \pm \infty}\left(\hat{U}_{t} \psi, f(x / t) \hat{U}_{t} \psi\right) \\
& =\lim _{t \rightarrow \pm \infty}\left(U_{t} \Omega_{ \pm}^{-1} \psi, f(x / t) U_{t} \Omega_{ \pm} \psi\right) \\
& \left.=\int d^{v} p \mid \widetilde{\left(\Omega_{ \pm}^{-1}\right.} \psi\right)\left.(p)\right|^{2} f(\nabla \omega(p))
\end{aligned}
$$

and the initial and final spatial distributions of $\psi_{t}$ are determined by the momentum distributions $\overparen{\Omega_{-}^{-1} \psi}$ and $\overparen{\Omega_{+}^{-1} \psi}$.

\section{The RAG theorem}

Our aim is to characterize the subspaces associated with the point spectrum and the continuous spectrum of a self-adjoint operator on $L^{2}\left(\mathbf{R}^{v}\right)$ in terms of "spacetime" properties. Ruelle [9] was the first to obtain results of this kind and his work was subsequently generalized by Amrein and Georgescu [1]. The basic idea behind both these works was an adaptation of the characterizations of almost periodic vectors and flight vectors in ergodic theory. In contrast, our discussion is based solely on the mean ergodic theorem in a form first stated by Wiener.

Let $t \in \mathbf{R} \rightarrow U_{t}$ be a strongly continuous one-parameter group of unitary operators on a Hilbert space $\mathscr{H}$. Further let $H$ denote the infinitesimal generator of $U$. The mean ergodic theorem states that

$$
\lim _{T \rightarrow \infty} \frac{1}{T} \int_{0}^{T} d t\left(\phi, U_{t} \psi\right)=\left(\phi, E_{H}(\{0\}) \psi\right)
$$

for all $\phi, \psi \in \mathscr{H}$ where $E_{H}$ denotes the spectral family associated with $H$. Wiener's theorem states that

$$
\lim _{T \rightarrow \infty} \frac{1}{T} \int_{0}^{T} d t\left|\left(\phi, U_{t} \psi\right)\right|^{2}=\sum_{\gamma \in \sigma_{p}(H)}\left|\left(\phi, E_{H}(\{\gamma\}) \psi\right)\right|^{2}
$$


for all $\phi, \psi \in \mathscr{H}$ where $\sigma_{p}(H)$ denotes the point spectrum of $H$. Although Wiener's theorem appears quite different to the mean ergodic theorem it is an immediate consequence of applying this result to the unitary group $U \otimes \bar{U}^{*}$ on the space $\mathscr{H} \otimes \overline{\mathscr{H}}\left(\overline{\mathscr{H}}\right.$ denotes the conjugate to $\mathscr{H}$ and $\left.\bar{U}_{t} \bar{\phi}=\overline{U_{t}^{*} \phi}\right)$. Our principal result is an extension of Wiener's theorem.

THEOREM 2. Let $A$ be a bounded operator on $\mathscr{H}$ and assume there exists a function $x \in \mathbf{R} \rightarrow f(x) \in \mathbf{C}$ such that

1. $f(H)$ is densely defined with a bounded inverse $f(H)^{-1}$;

2. $A f(H)^{-1}$ is compact.

It follows that

$$
\lim _{T \rightarrow \infty} \frac{1}{T} \int_{0}^{T} d t\left\|A U_{t} \psi\right\|^{2}=\sum_{\gamma \in \sigma_{p}(H)}\left\|A E_{H}(\{\gamma\}) \psi\right\|^{2} .
$$

Proof. Since both sides of the required relation are continuous in $\psi$ it suffices to derive the relation for $\psi$ in the dense subset formed by the domain $D(f(H))$ of $f(H)$. But, if $\psi \in D(f(H))$, then $\psi=f^{\prime}(H)^{-1} \phi$ for some $\phi$ and

$$
\begin{aligned}
\left\|A U_{t} \psi\right\|^{2} & =\left\|A U_{t} f(H)^{-1} \phi\right\|^{2} \\
& =\left\|A f(H)^{-1} U_{t} \phi\right\|^{2} .
\end{aligned}
$$

Next, consider the positive compact operator

$$
C=\left(A f(H)^{-1}\right)^{*}\left(A f(H)^{-1}\right) .
$$

For each $\varepsilon>0$ there are $\lambda_{i} \geqslant 0$ and $\phi_{i} \in \mathscr{H}$, such that

$$
\left\|C-\sum_{i=1}^{n} \lambda_{i} E_{\phi_{i}}\right\|<\varepsilon
$$

where $E_{\phi_{i}}$ is the rank one projector with $\phi_{i}$ in its range. Thus

$$
\left.\left|\left\|A U_{t} \psi\right\|^{2}-\sum_{i=1}^{n} \hat{\lambda}_{i}\right|\left(\phi_{i}, U_{t} \phi\right)\right|^{2} \mid<\varepsilon .
$$

Now applying Wiener's theorem one obtains

$$
\left|\lim _{T \rightarrow \infty} \frac{1}{T} \int_{0}^{T} d t\left\|A U_{t} \psi\right\|^{2}-\sum_{\gamma \in \sigma_{p}(H)} \sum_{i=1}^{n} \lambda_{i}\right|\left|\left(\phi_{i}, E_{H}(\{\gamma\}) \phi\right)\right|^{2} \mid<\varepsilon .
$$

Reapplying the approximation for $C$ then gives

$$
\left|\lim _{T \rightarrow \infty} \frac{1}{T} \int_{0}^{T} d t\left\|A U_{\imath} \psi\right\|^{2}-\sum_{\gamma \in \sigma_{p}(H)}\left\|A f(H)^{-1} E_{H}(\{\gamma\}) \phi\right\|^{2}\right|<2 \varepsilon .
$$


But $f(H)^{-1}$ commutes with $E_{H}$ and hence

$$
\left|\lim _{T \rightarrow \infty} \frac{1}{T} \int_{0}^{T} d t\left\|A U_{t} \psi\right\|^{2}-\sum_{\gamma \in \sigma_{p}(H)}\left\|A E_{H}(\{\gamma\}) \psi\right\|^{2}\right|<2 \varepsilon .
$$

Since $\varepsilon>0$ was arbitrary this completes the proof.

Next let $\mathscr{H}_{p}$ and $\mathscr{H}_{c}$ denote the closed subspaces of $\mathscr{H}$ corresponding to the point spectrum and the continuous spectrum of $H$ respectively. Thus

$$
\mathscr{H}=\mathscr{H}_{p} \oplus \mathscr{H}_{c} .
$$

COROLlary 1. Under the conditions of Theorem 1 with $A$ invertible, the following conditions are equivalent:

1. $\psi \in \mathscr{H}_{c}$.

2. $\lim _{r \rightarrow \infty} \frac{1}{T} \int_{0}^{T} d t\left\|A U_{t} \psi\right\|^{2}=0$.

This is an immediate consequence of Theorem 2 and the relation $\mathscr{H}_{c}=\mathscr{H}_{p}^{\perp}$.

The case of greatest interest for quantum mechanical scattering theory is $\mathscr{H}=L^{2}\left(\mathbf{R}^{v}\right)$ and $A=\chi_{R}$ is the operator of multiplication by the characteristic function of the ball of radius $R$ in $\mathbf{R}^{\nu}$.

COROLLARY 2. Assume there exists a function $x \in \mathbf{R} \rightarrow f(x) \in C$ such that

1. $f(H)$ is a densely defined operator with bounded inverse $f(H)^{-1}$,

2. $\chi_{R} f(H)^{-1}$ is compact for all $R \geqslant 0$.

It follows that

$$
\lim _{R \rightarrow \infty} \lim _{T \rightarrow \infty} \frac{1}{T} \int_{0}^{T} d t\left\|\chi_{R} U_{t} \psi\right\|^{2}=\left\|E_{p}(H) \psi\right\|^{2},
$$

where $E_{p}(H)$ is the projector onto $\mathscr{H}_{p}$. Therefore $\psi \in \mathscr{H}_{p}$ if, and only if,

$$
\lim _{R \rightarrow \infty} \lim _{T \rightarrow \infty} \frac{1}{T} \int_{0}^{T} d t \int_{|x| \leqslant R} d^{v} x\left|\left(U_{t} \psi\right)(x)\right|^{2} /\|\psi\|^{2}=1,
$$

and $\psi \in \mathscr{H}_{c}$ if, and only if,

$$
\lim _{R \rightarrow \infty} \lim _{T \rightarrow \infty} \frac{1}{T} \int_{0}^{T} d t \int_{|x| \leqslant R} d^{v} x\left|\left(U_{t} \psi\right)(x)\right|^{2}=0 .
$$

Proof. One may apply Theorem 2 with $A=\chi_{R}$ and hence

$$
\lim _{T \rightarrow \infty} \frac{1}{T} \int_{0}^{T} d t\left\|\chi_{R} U_{t} \psi\right\|^{2}=\sum_{\gamma \in \sigma_{p}(H)} \| \chi_{R} E_{H}\left(\left\{\gamma\{) \psi \|^{2} .\right.\right.
$$


But

and

$$
\begin{aligned}
\sum_{\gamma \in \sigma_{p}(H)}\left\|\chi_{R} E_{H}(\{\gamma\}) \psi\right\|^{2} & \leqslant \sum_{\gamma \in \sigma_{p}(H)}\left\|E_{H}(\{\gamma\}) \psi\right\|^{2} \\
& =\left\|E_{p}(H) \psi\right\|^{2}
\end{aligned}
$$

Thus

$$
\lim _{R \rightarrow \infty}\left\|\chi_{R} E_{u}(\{y\}) \psi\right\|=\left\|E_{n}(\{y\}) \psi\right\|
$$

$$
\begin{aligned}
\lim _{R \rightarrow \infty} \lim _{T \rightarrow \infty} \frac{1}{T} \int_{0}^{T} d t\left\|\chi_{R} U_{t} \psi\right\|^{2} & =\sum_{\gamma \in \sigma_{p}(H)} \lim _{R \rightarrow \infty}\left\|\chi_{R} E_{H}(\{\gamma\}) \psi\right\|^{2} \\
& =\left\|E_{p}(H) \psi\right\|^{2}
\end{aligned}
$$

by the Lebesgue dominated convergence theorem. The characterizations of $\mathscr{H}_{\mathrm{p}}$ and $\mathscr{H}_{c}$ follow immediately.

REMARK. Since $R \rightarrow\left\|\chi_{R} U_{t} \psi\right\|^{2}$ is a monotonically increasing function, the criterion for $\mathscr{H}_{c}$ may be restated as $\psi \in \mathscr{H}_{c}$ if, and only if,

for all $R \geqslant 0$, that is,

$$
\lim _{T \rightarrow \infty} \frac{1}{T} \int_{0}^{T} d t\left\|\chi_{R} U_{t} \psi\right\|^{2}=0
$$

$$
\lim _{T \rightarrow \infty} \frac{1}{T} \int_{0}^{T} d t \int_{|x| \leqslant R} d^{v} x\left|\left(U_{t} \psi\right)(x)\right|^{2}=0
$$

for all $R \geqslant 0$. This is Ruelle's result [9]. Similarly, the characterization of $\mathscr{H}_{p}$ may be restated as $\psi \in \mathscr{H}_{p}$ if, and only if, for $\varepsilon>0$ there is an $R>0$ such that

$$
\lim _{T \rightarrow \infty} \frac{1}{T} \int_{0}^{T} d t \int_{|x| \geqslant R} d^{\mathrm{v}} x\left|\left(U_{t} \psi\right)(x)\right|^{2}<\varepsilon .
$$

Ruelle [9] showed that the mean value is not necessary in this last formula, that is, $\psi \in \mathscr{H}_{p}$ implies that

$$
\int_{|x| \geqslant R} d^{v} x|(U, \psi)(x)|^{2}<\varepsilon
$$

for $R$ sufficiently large, uniformly in $t$.

The assumptions of Corollary 2 can be verified in many cases of interest.

Case 1. Let $H=H_{0}$ where $H_{0}$ is an operator of multiplication on the Fourier space

$$
\left(H_{0} \tilde{\psi}\right)(P)=\omega(p) \tilde{\psi}(p)
$$


and $\omega$ is a real Lebesgue measurable function satisfying $|\omega(p)| \rightarrow \infty$ as $|p| \rightarrow \infty$. It follows that $\chi_{R}\left(H_{0} \pm i\right)^{-1}$ is compact.

Case 2. Let $V$ be a self-adjoint operator such that $H=H_{0}+V$ is self-adjoint on $D\left(H_{0}\right) \cap D(V)\left(H_{0}\right.$ as in 1$)$; then $\chi_{R}(H \pm i)^{-1}$ is compact.

Case 3. Let $W$ be a self-adjoint operator such that the form sum $H=H_{0}+V+W$ is self-adjoint $\left(H_{0}+V\right.$ as in 2$)$; then $\chi_{R}(H \pm i)^{-1}$ is compact.

(For proofs of these statements see [1] where a variety of other cases are also handled.)

Corollary 2 has an obvious physical interpretation in terms of bound states and non-bound states. It concludes that $\psi \in \mathscr{H}_{p}$ if and only if the corresponding quantum mechanical particle remains close to the origin. Thus $\mathscr{H}_{p}$ corresponds to the subspace of bound states and its orthogonal complement $\mathscr{H}_{c}$ corresponds to unbound, or scattering, states.

Although this discussion is only directly applicable to the relative motion of two particles it can be extended to the full motion as follows. The appropriate Hilbert space is $\mathscr{H}=L^{2}\left(\mathbf{R}^{v}\right) \otimes L^{2}\left(\mathbf{R}^{v}\right)$ where the tensor product is symmetrized, or antisymmetrized, if the particles are identical Bosons, or Fermions. The values $\psi\left(x_{1}, x_{2}\right)$ of each $\psi \in \mathscr{H}$ describe the quantum-mechanical distribution of a particle at the point $x_{1}$ and a second particle at the point $x_{2}$. But there is a second factorization $\mathscr{H}=\mathscr{H}^{\mathrm{cm}} \otimes \mathscr{H}^{\text {rel }}$ corresponding to the choice of centre of mass and relative coordinates

$$
X=\frac{1}{2}\left(x_{1}+x_{2}\right), \quad x=x_{1}-x_{2} .
$$

In this second factorization $\mathscr{H}^{\mathrm{cm}}$ is isomorphic to $L^{2}\left(\mathbf{R}^{v}\right)$ and $\mathscr{H}^{\text {rel }}$ is isomorphic to $L^{2}\left(\mathbf{R}^{v}\right)$ or the subspace of symmetric, or anti-symmetric, functions. If the particles interact through a translationally invariant two-body potential the total Hamiltonian $H$ separates with respect to this second factorization in the form

$$
H=H^{\mathrm{cm}} \otimes \mathbf{I}+\mathbf{I} \otimes H^{\mathrm{rel}},
$$

where $H^{\mathrm{cm}}$ is a sub-Hamiltonian corresponding to the centre of mass motion and $H^{\text {rel }}$ describes the relative motion. Since $H^{\mathrm{cm}}$ has continuous spectrum the total Hamiltonian has continuous spectrum. Nevertheless there is a factorization of $\mathscr{H}$ corresponding to the point spectrum and continuous spectrum of $H^{\text {rel }}$. Let $\mathscr{H}_{p}^{\text {rel }}, \mathscr{H}_{c}^{\text {rel }} \subseteq \mathscr{H}^{\text {rel }}$ denote the subspaces of point, and continuous, spectrum of $H^{\text {rel }}$ and define

$$
\mathscr{H}_{p}=\mathscr{H}^{\mathrm{cm}} \otimes \mathscr{H}_{p}^{\mathrm{rel}}, \quad \mathscr{H}_{\mathrm{c}}=\mathscr{H}^{\mathrm{cm}} \otimes \mathscr{H}_{c}^{\mathrm{rel}} .
$$

One can now characterize elements of $\mathscr{H}_{p}$ and $\mathscr{H}_{c}$ by criteria of the type given in Corollary 2. This is based on an extension of Theorem 2 . 
COROllaRy 3. Let $\mathscr{H}=\mathscr{H}_{1} \otimes \mathscr{H}_{2}$ and assume that the generator $H$ of $U_{t}$ has the form $H=H_{1} \otimes \mathbf{I}+\mathbf{I} \otimes H_{2}$ where $H_{1}$ and $H_{2}$ are self-adjoint operators on $\mathscr{H}_{1}$ and $\mathscr{H}_{2}$ respectively. Let $A$ be a bounded operator on $\mathscr{H}_{2}$ and assume that there exists a function $x \in \mathbf{R} \rightarrow f(x) \in \mathbf{C}$ such that

1. $f\left(\mathrm{H}_{2}\right)$ is densely defined with bounded inverse $f\left(\mathrm{H}_{2}\right)^{-1}$;

2. $A f\left(H_{2}\right)^{-1}$ is compact.

It follows that

$$
\lim _{T \rightarrow \infty} \frac{1}{T} \int_{0}^{T} d t\left\|(\mathbf{I} \otimes A) U_{t} \psi\right\|^{2}=\sum_{\gamma \in \sigma_{p}\left(H_{2}\right)}\left\|\left(\mathbf{I} \otimes A E_{H_{2}}(\{\gamma\})\right) \psi\right\|^{2} .
$$

The proof is essentially identical to that of Theorem 2 except one must also use a strong approximation of $\psi$ by a sum $\sum_{i} \lambda_{i} \psi_{i}^{(1)} \otimes \psi_{i}^{(2)}$ of products of orthonormal vectors $\psi_{i}^{(1)} \in \mathscr{H}_{1}$, and $\psi_{i}^{(2)} \in D\left(f\left(H_{2}\right)\right)$. We omit the details.

Therefore, under assumptions on the relative Hamiltonian identical to those of Corollary 2 , one finds $\psi \in \mathscr{H}_{p}$ if, and only if,

$$
\lim _{R \rightarrow \infty} \lim _{T \rightarrow \infty} \frac{1}{T} \int_{0}^{T} d t \int_{\left|x_{1}-x_{2}\right| \leqslant R} d x_{1} d x_{2}\left|\left(U_{t} \psi\right)\left(x_{1}, x_{2}\right)\right|^{2} /\|\psi\|^{2}=1,
$$

and $\psi \in \mathscr{H}_{c}$ if, and only if,

$$
\lim _{R \rightarrow \infty} \lim _{T \rightarrow \infty} \frac{1}{T} \int_{0}^{T} d t \int_{\left|x_{1}-x_{2}\right| \leqslant R} d x_{1} d x_{2}\left|\left(U_{t} \psi\right)\left(x_{1}, x_{2}\right)\right|^{2}=0 .
$$

These methods also yield characterizations of the bound states of three or more particles and it should be possible to characterize the subspaces describing bound states of subsets of the particles. But this no doubt requires more stringent assumptions on the range of the interparticle interaction.

\section{References}

[1] W. O. Amrein and V. Georgescu, "Bound states and scattering states in quantum mechanics", Helv. Phys. Acta. 46 (1973), 635-658.

[2] J. M. Combes, R. G. Newton and R. Shtokhamer, "Scattering into cones and flux across surfaces", Phys. Rev. D. 11, 2 (1975), 366-372.

[3] J. D. Dollard, "Scattering into cones I: Potential scattering", Commun. Math. Phys. 12 (1969), 193-203.

[4] T. A. Green and O. E. Lanford III, "Rigorous derivation of the phase shift formula for the Hilbert space scattering operator of a single particle", J. Math. Phys. 1 (1960), 139-148.

[5] J. M. Jauch, R. Lavine and R. G. Newton, "Scattering into cones", Helv. Phys. Acta. 45 (1972), 325-330.

[6] T. Kato, Perturbation theory for linear operators (Springer-Verlag, 1966).

[7] R. G. Newton and R. Shtokhamer, "N-particle scattering rates", in Physical reality and mathematical description (ed. C. P. Enz and J. Mehra) (Reidel, New York, 1974). 
[8] M. Reed and B. Simon, Methods of modern mathematical physics, Vol. I (Academic Press, New York, 1972).

[9] D. Ruelle, "A remark on bound states in potential scattering theory", Nuovo Cim. 61A (1969), 655-662.

School of Mathematics

University of New South Wales

Kensington

NSW 2033 